\title{
Topology Control in Mobile Wireless Sensor Networks
}

\author{
Umut Can Çabuk*1, Gökhan Dalkılıç ${ }^{2}$
}

Accepted $3^{\text {rd }}$ September 2016

\begin{abstract}
Topology control is a set of strategies and actions those aim to improve energy efficiency, lifetime and reliability of the networks. One of the most effective methodology is topology reduction, which is a part of the overall topology control process. Topology control process is usually considered under two phases: construction and maintenance. This hard work would even be harder for mobile ad-hoc sensor networks, those consist of devices with limited capabilities and continual independent movement. In this work, we made our efforts to find out effects of mobility of the nodes in an ad-hoc wireless sensor network on network parameters, by using different pre-defined topology control protocols. Thus, we made simulations using an experimental simulator, called Atarraya. Furthermore, we measured the performance of each protocol and compared the outcomes in order to find the best topology control strategy. Lastly, possible solutions to the uncovered problems are proposed.
\end{abstract}

Keywords: Ad-hoc wireless sensor networks, mobility, topology control, energy efficiency, simulation.

\section{Introduction}

After conventional wide area and local area networks (LAN), nowadays, personal area (PAN) and body area networks (BAN) are becoming more and more popular in applications of industry, commerce and science [1]. Along with their abilities and advantages, these networks also have their own challenges [2]. For instance, they usually include nodes on the move or at least nodes that can be relocated. Hence, the newest solutions in the interconnecting network technologies have to deal with the mobility cases as well.

From that starting point, in order to build the rest of the work on it, we've presumed a set of use cases, called as scenario. Although the results wouldn't be directly related to the scenario, it would help us to make some decisions. Our scenario based on an indoor working environment including unsynchronized moving people, who are representing BAN or PAN networks by holding or even wearing wireless sensor devices. Most applicable realization is hospitals, but also mines, schools, prisons, sport fields and in a wider point of view, zoos. Additionally, we suppose a continuous communication is required in our scenario.

\section{Previous Works}

While building our background, we found out several studies about topology control [3] and maintenance [4] in wireless sensor networks. And of course, we used valuable information provided in these works. But we couldn't find a comparative study to refer, which focuses directly on mobility case. For instance, in [5] authors present a comparative study similar to ours, however they used fixed nodes and totally excluded the mobility case, which is our main focus. In their work, Latré et al. [6] introduce a protocol (Cascading information retrieval by controlling access with distributed slot assignment - CICADA) designed for mobility cases and present a detailed analysis, but their study is not

\footnotetext{
${ }^{1}$ Department of Electrical and Electronics Engineering, Erzincan

University, Yalnizbag Campus, Erzincan, Turkey

${ }^{2}$ Department of Computer Engineering, Dokuz Eylul University, Tinaztepe Campus, Izmir, Turkey

* Corresponding Author: Email: ucabuk@erzincan.edu.tr

Note: This paper has been presented at the $3^{\text {rd }}$ International Conference on Advanced Technology \& Sciences (ICAT'16) held in Konya (Turkey), September 01-03, 2016.
}

comparative. Reluctantly, we could not include CICADA and CICADA-S (a secure version proposed later [7]) in our work because of the limitations of the tool we have used.

The reason of that scarcity is that wireless sensor networks is a quite new area, and mobility in these networks is even a newer aspect. Most of the previous works in this area focused on higher layer architecture, like routing and forwarding protocols. Here, we worked on a lower level relationship between nodes by reducing the number of links between them.

The simulator we used, namely Atarraya, was also introduced in a previous work [8] and still under development with new features. A side note: Mobility wasn't even included in our simulation tool Atarraya's first version, here we used version 1.3.

\section{Assumptions \& Decisions}

To find key points, we had to choose optimum preferences and had to limit our angle of sight. Our very first assumption about the concept is a scenario as described in introduction. Before going on, we assume the undetermined smallest integer time unit in Atarraya as milliseconds.

\subsection{Algorithms}

For the evaluation, we wanted to choose topology control and maintenance algorithms, which have different approaches as possible and tried to avoid similar algorithms. So, we have chosen A3, energy efficient connected dominating set (EECDS) and Kneigh tree protocols for topology construction. Detailed descriptions of these protocols can be found in $[9,10]$. Then we considered dynamic global time-based topology recreation (DGTTRec), static global time-based topology rotation (SGTTRot), and HGTTRec and rotation (HGTTRecRot) for topology maintenance. All these algorithms are chosen from the set that is predefined in the tool [8]. Adding custom protocols is not possible for end-users but the source of the tool must be edited, which could be done in another work.

During topology maintenance evaluation, we used A3 as a base construction algorithm, in order to make results comparable.

Energy efficiency is always a fundamental aim, but in mobile networks geographic distribution of nodes is dynamic, this means physical link between nodes are not stable, hence it is more accurate to use time triggered maintenance algorithms than energy threshold triggered ones, to be sure about the connectivity. 


\subsection{Test Scene, Parameters \& Metrics}

We created a $1000 \times 1000 \mathrm{~m}^{2}$ observation field, but didn't use this entire field for initial node deployment. We placed 30 nodes in a $500 \times 500 \mathrm{~m}^{2}$ area in the center of the observation field mentioned above, using Normal distribution with sigma $=100$. When simulation starts, nodes can leave this initial deployment area and can move in the whole observation field, or even may leave that area.

Our test field represents a surrounded area like a hospital, a factory or an office environment. It can also be an open-air field but we mostly focused on an indoor environment. Hence, we placed 30 nodes ( +1 initializer sink), which makes a sparse network. This number may be increased until around 100 without distorting the test outcomes. Numbers bigger than a few hundred may cause the simulator to slow down, stutter or act inconsistently.

We used a realistic communication range of $100 \mathrm{~m}$, which is typical for ZigBee, Bluetooth Class 1 and Wi-Fi b/g/n [11]. We set sensing area to $20 \mathrm{~m}$ but, in this work, it wasn't important in our point of view, so we didn't take care of sensing range and sensed data.

As total simulation time for topology maintenance tests, we considered 60 seconds. It was enough to see effects of mobility, plus we had some limitations about our movement model (see below). This interval theoretically provides $600 \mathrm{~m}$ of moving range to each node (see below). Thus, we also decided re-setting the topology time interval $1 \mathrm{~s}$.

We assigned $1000 \mathrm{~mJ}$ total energy stock to each node and used simple energy model of the software. We also used simple communication model. Another assumption was 0 bit error rate (BER) value, means communication link is perfect. Effect of BER is subsequently analyzed.

Finally, a connectivity metric is defined to measure the performance of the protocols in the sense of physical and logical connectivity. This metric is considered as a percentage of nodes (directly or as multi-hop) connected to the sink node among all the nodes in the coverage of the core network. Basically, the nodes out of the core network coverage are excluded from that ratio. Here, core network means the network initialized by the sink.

$$
C(\%)=\frac{\# \text { of nodes connected to network }(t)-1}{\# \text { of nodes in the range of network }(t)-1}
$$

Obviously, this connectivity metric is a function of time (and movement), so it will also represent robustness and sustainability of the protocols. By the way, this metric can be the most important information to determine the usefulness of a topology control protocol depending on the scenario.

\subsection{Movement Model}

We designed a movement model in order to provide mobility to nodes in the simulation. There are two main limitations to consider: First, movement model should be realistic, thus results will be useful in further works and industrial applications. Second, simulation tool Atarraya is an (very useful but) experimental tool and has limited capabilities in this manner [8]. So, our movement model consists of these features: Simple random walk mechanism for each node with sleep time $100 \mathrm{~ms}$ and step size $1 \mathrm{~m}$, without surrounding limits (fences) on the field. This makes $10 \mathrm{~m} / \mathrm{s}$ and is 5 to 10 times faster than the average human walking speed, which is around 1 to $2 \mathrm{~m} / \mathrm{s}$ [12]. We used that increased value to see the effects of mobility more clearly. By using randomness, movement of nodes is nondeterministic to each other and no predictions can be made besides the step size.
Another characteristic of the model is spreading. All nodes in a very long period of time, eventually, will leave the test field since there is no fence. This is not an intended behavior but due to program limitations, it is not possible to prevent this. Only the simulation time can be limited at an optimal time period. However, this behavior allows us to see what happens when some nodes leave the area, which can be a realistic scenario.

\section{Results}

We first evaluated the topology construction protocols, by starting the simulation without a time limit. 30 Nodes and 1 sink node are deployed in the field as described above. Simulation ended up after a successful topology creation. All values given in this section are average of 10 measurements.

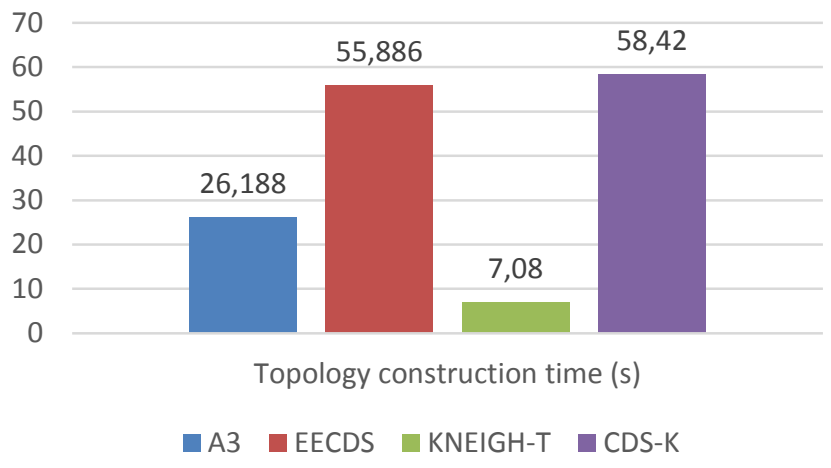

Figure 1. Durations of the initial topology construction period.

Figure 1 gives the time passed until the successful creation for each protocol. Here, it is very clear that, tree based A3 and Kneigh tree protocols are much faster than dominating set based EECDS and k-connected k-dominating set (CDS-K). Due to the mobility of the nodes, it would be better to use faster protocols.

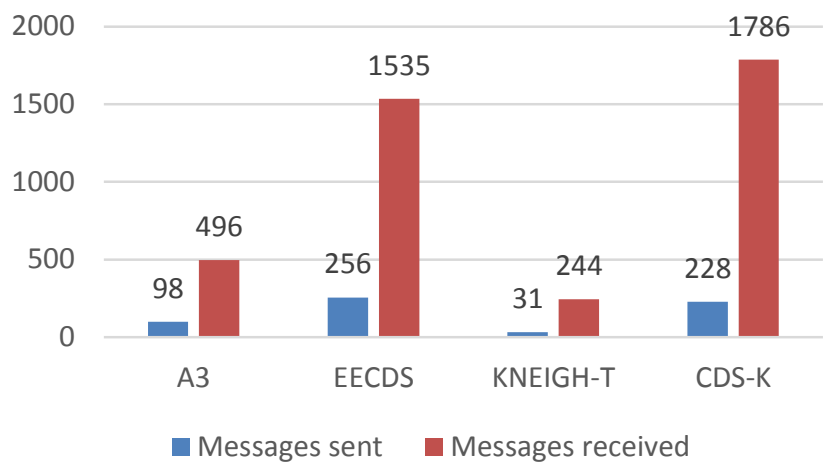

Figure 2. Total message traffics during the initial topology construction.

Total message traffic can be seen in Figure 2, which stands for the total number of sent and received messages in the network, between all 31 nodes. Here, A3 and Kneigh tree produce significantly less traffic than EECDS and CDS-K. Lower values would be preferable because sending and receiving more messages will cost more energy, processing power and bandwidth.

Figure 3 shows total energy consumed among whole network. These values are some of the consumptions of all 31 nodes. As expected from the message traffic results, A3 and Kneigh tree consume much less energy from EECDS and CDS-K. While Kneigh tree consumes least energy, EECDS becomes the last preferable with the highest consumption, which is 4 times more than $\mathrm{A} 3$ and 30 times more than Kneigh tree. 
A very important statement; all these protocols complete their work by constructing the topology much faster than the movement speed of mobile nodes. Our movement model proposes a $5 \mathrm{~m}$ step size per $500 \mathrm{~ms}$. But, here the slowest protocol, CDS-K, requires $58.42 \mathrm{~ms}$. Even when we change our movement model (unless we set an unrealistic super speed like $100 \mathrm{~m}$ step size per $10 \mathrm{~ms}$ or so), the results are (almost) the same as stationary ad-hoc wireless sensor networks' results.

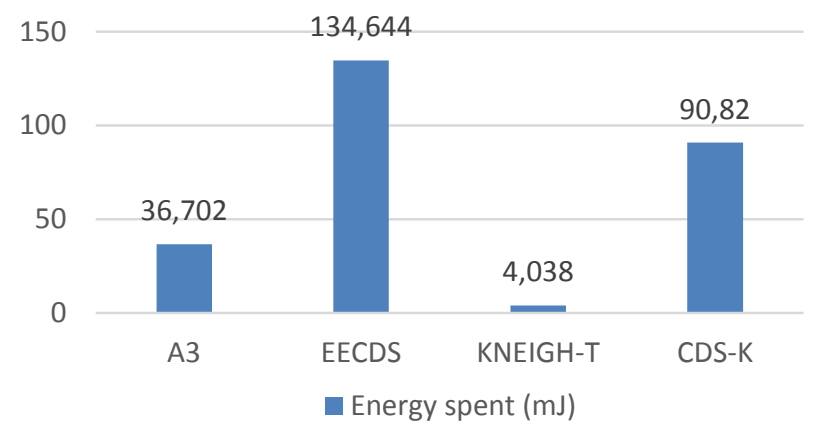

Figure 3. Total energy consumptions in the whole network during topology construction (for 31 nodes)

So, information given until here, are not enough to uncover the effects of the mobility in that network. Because, if there were no mobility, results would be very close, and also network operating time should be much higher than construction time. Over and above, we have to check topology maintenance simulation results.

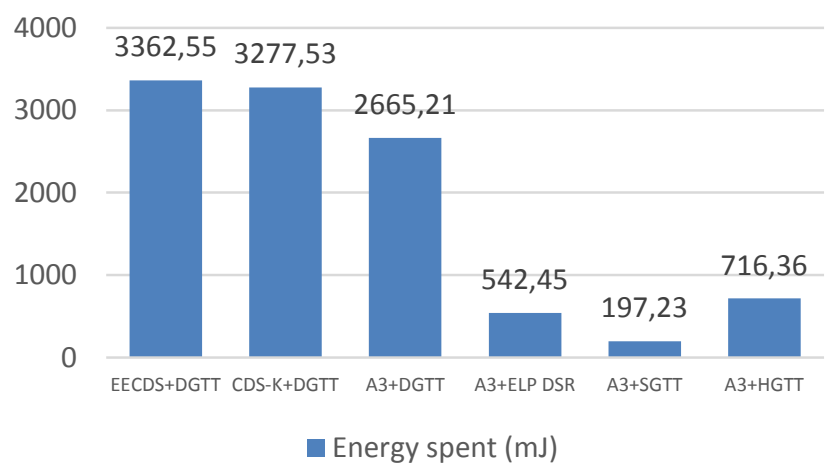

Figure 4. Total energy consumptions in the whole network during topology maintenance in 1 minute run (for 31 nodes).

Figure 4 represents total amount of energy consumed by all 31 nodes during 1 minute run of the protocols. Here we can easily say DGTT consumes 5, 12 and 3.5 times more energy than DSR, SGTT and HGTT respectively. Among the DGTT experiments, most energy-efficient choice is A3. This result is reasonable, because, from the previous experiments (Figure 3) we found out that $\mathrm{A} 3$ is more effective than EECDS and CDS Rule K, moreover, ECDS and CDS-K were very close. Here the same.

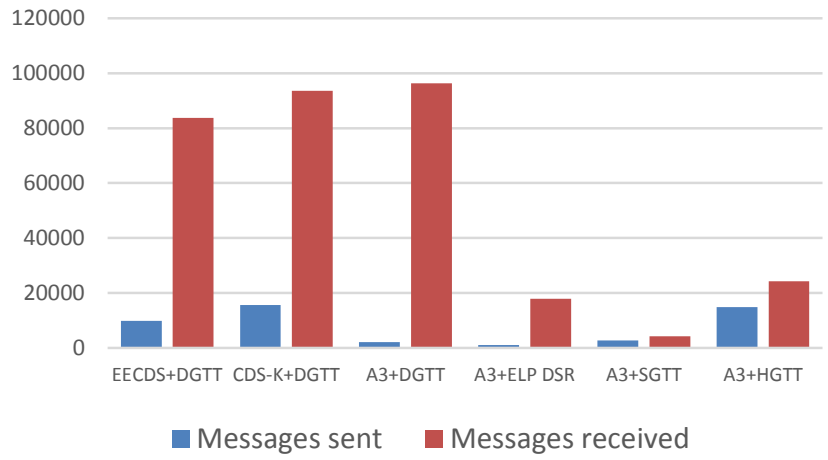

Figure 5. Total message traffic during topology maintenance in 1 minute.

In Figure 5, total message traffic of the network is given. There is a huge difference between DGTT and the others. Actually, the number of sent messages is very similar, just HGTT is relatively higher. But, the number of received messages is very high in DGTT. It is 4 times higher than the runner-up HGTT. SGTT has the lowest total traffic in general. In fact, the reason of this big difference between the number of received and sent messages is broadcast style transmission and mass reception of the broadcasted messages by all nodes in the range. This behavior artificially boosts the network traffic.

Probably the most important data is presented in Figure 6: connectivity metric. It shows reliability and sustainability of the protocols via 2 different time intervals. Here, all DGTT combinations together get the highest value by $\% 100$ connectivity rate in both 1-minute and 5-minutes tests. Mobility (in the coverage area of course) does not cause impairment while using DGTT. Distinctly, if a node is in the communication range, then it will be included to the network via DGTT. The others have relatively lower values in 1-minute test and they become much worse by the time, as can be seen in 5-minutes test. In a 10minutes test (which is not shown in the figure) ratios of SGTT and HGTT are below $25 \%$.

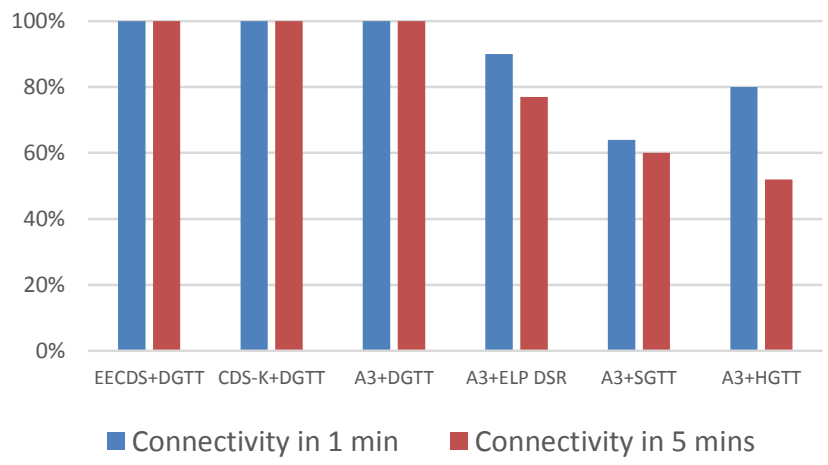

Figure 6. Connectivity levels during topology maintenance in 1 minute and 5 minutes of run.

Manifestly, here, we discovered a clear tradeoff between energy spent plus message traffic and connectivity performance. There is no linearity, but the correlation is clear. Before evaluating the values given in Figure 6, it wasn't possible to say A3 or CDS-K or even EECDS can be a good choice. But after, we can say, for that type of a network, DGTT has the best results and preferable with A3, from the previous results.

So-called energy efficiency and traffic emptiness of SGTT and HGTT can be very deceptive. This is highly probable a paralogy, caused by the low connectivity and the link loss. Less links mean, less message receptions and less message receptions lead less energy consumption. Even so, this conjecture is valid for specific scenarios and could be different under variety of conditions. 
All in all, although these values can give very valuable information and can provide guidance for related applications, the optimum decision is always application dependent. For instance, in a scenario that continuous communication is not as important as node lifetime (energy efficiency), DGTT should be avoided, while A3 could still be preserved.

We also evaluated the effect of BER on DGTT with A3, CDS-K and EECDS respectively. In Figure 7, the relation between BER and connectivity is analyzed. It can be clearly seen that, a higher error rate lowers the connectivity metric significantly, but this relation is roughly logarithmical. While the error rate is $10^{\wedge}-5$, which is a de facto industry standard boundary, connectivity metric is very high; $97 \%$ to $100 \%$. And until $10^{\wedge}-3$ values are acceptable. But one shouldn't forget that, sensor data transfer is not included in this communication. It just represents topology control messagings. So, for higher level decisions about the architecture, level of the BER should be maintained no lower than $10^{\wedge}-5$ if the data sensed is time critical.

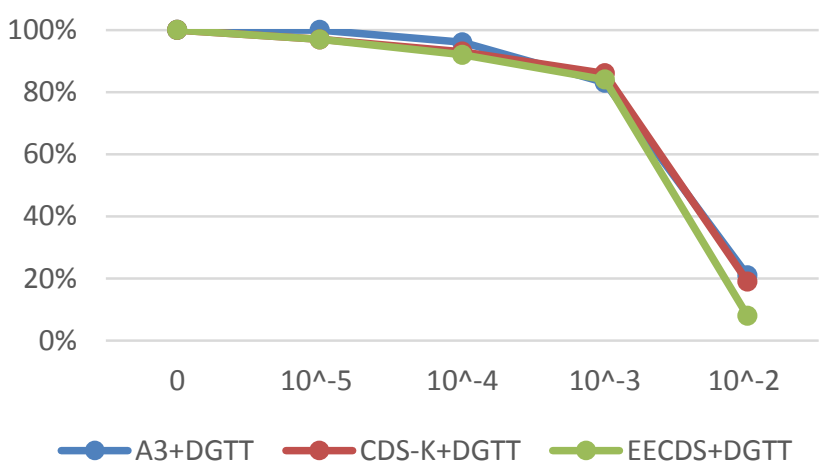

Figure 7. Effect of the bit error rate on the connectivity ratio.

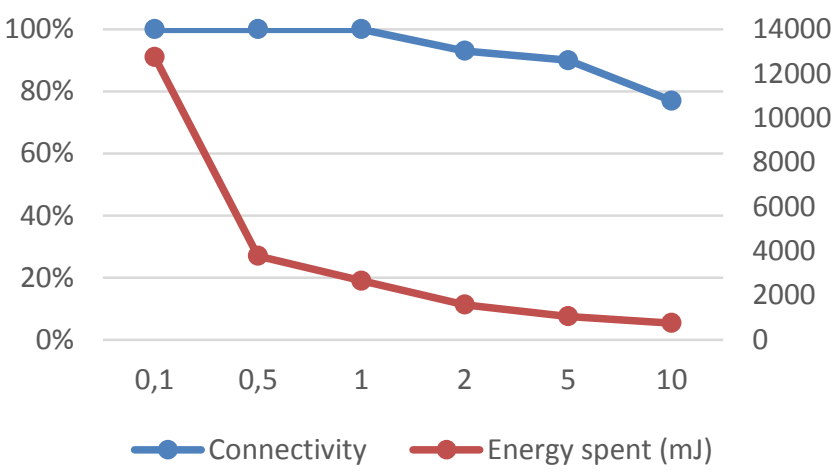

Figure 8. Effect of topology recreation time interval on connectivity and energy consumption based on 1 minute run using A3 + DGTT.

We discovered a clear correlation between connectivity and energy consumption, but this does not mean there is nothing to do to save energy. Figure 8 shows the effect of topology recreation interval during maintenance on both connectivity and energy consumption. First, please note that we used 500 ms movement sleep time and $1 \mathrm{~s}$ refreshing interval in previous experiments. Here we kept movement model as defined, but tested different time intervals. Hence, we exposed that, a refreshing frequency which is much higher than the movement speed (this also means physical deployment change frequency) causes extraordinary energy consumption and highly ineffective. We can also see that a frequency much lower, causes loss of communication links. Thus, a balance should be preserved to save energy and to protect connectivity at the same time. An interval 2 to 4 times longer than basic movement period looks acceptable in our case. As a final comment, statistical information about the deployment environment and movement should be obtained to find a (sub-) optimal solution.

\section{Conclusions \& Future Works}

In this work, we aimed to show possible effects of the mobility to ad-hoc network performance and reliability in different aspects. Furthermore, we wanted to propose basic solutions.

In spite of the application dependability, we got some solid outcomes; first, unlike DGTT, SGTT and HGTT are not suitable for that type of a mobile network. One should better prefer a dynamic protocol, at least in a similar scenario like our presumption. Because of the connectivity level provided, we are not satisfied with the performance of static and hybrid methods. But in a different scenario, significant energy saving (we also found out that this is deceptive and caused by physical link loss) of these protocols can be exploited in a way.

Additionally, between the construction algorithms, A3 provides better values from the rest apart from Kneigh tree. But in long term, we showed that this advantage gained from construction phase becomes less significant by the time. Even so, A3 could be a reasonable choice for our scenario.

An impromptu (but predictable) observation (or solution) to the "connectivity-energy efficiency" problem is also given.

We got a strong impression about; building a cell-like structure by placing several stationary nodes in the deployment field could help us to reduce redundant traffic and to increase energy efficiency without downgrading the link quality. This hybrid network structure may give better results. This strategy should be researched and it can be a good extension to this work.

We still have no overall optimum choices, so search for a topology maintenance protocol that offers a better balance between energy consumption (or message traffic) and connectivity should go on.

Another point is super-speed mobility. We created a realistic human-like movement model; it can also be used for animals, or robotic structures. But super-speed mobility is not supported; in that case, it is not even possible to construct a network. And even we deploy a cell structure, handovers would be a big problem. However, super-speed moving objects, including racing cars, satellites, missiles or so, rarely move randomly. They follow a track or orbit. This feature can be exploited. So, that aspect can also be another topic to investigate.

\section{Acknowledgements}

We want to thank Dr. Qi Zhang from Aarhus University, Department of Engineering, for her valuable guidance. Most work is done using the simulator Atarraya and we would like to present our sincerest respect and recognition to creators of the program: Pedro M. Wightman and Miguel A. Labrador.

\section{References}

[1] S. Gonzalez-Valenzuela et al., Autonomous Sensor Networks: Collective Sensing Strategies for Analytical Purposes, D. Filippini, Ed. Berlin, Germany: SpringerVerlag, pp. 17-38, 2012.

[2] M. A. Hanson, et al., "Body area sensor networks: Challenges and opportunities," Computer, vol. 42, no. 1, pp. 58-65, Jan. 2009.

[3] P. M. Wightman Rojas, "Topology Control in Wireless Sensor Networks," PhD thesis, Computer Science and Engineering Department, University of South Florida, Tampa, Florida, USA, 2010.

[4] P. M. Wightman, M. A. Labrador, "Topology Maintenance: Extending the Lifetime of Wireless Sensor Networks," IEEE Latin America Transactions, vol. 8, no. 4, pp. 469- 
475, Aug. 2010.

[5] G. Pachnanda, R. Chaudhary, "Comparative Study of A3, EECDS, CDS Rule $\mathrm{K}$ and KNEIGH Tree Protocols in a Grid Manner," Advances in Electronic and Electric Engineering, vol. 3, no. 4, pp. 509-514, 2013.

[6] B. Latré, et al., "A low-delay protocol for multihop wireless body area networks," in Proc. 4th Annual International Conference on Mobile and Ubiquitous Systems: Networking \& Services, 2007, pp. 1-8.

[7] D. Singelée, et al., "A Secure Low-Delay Protocol for Wireless Body Area Networks," Ad Hoc \& Sensor Wireless Networks, vol. 9, pp. 53-72, 2010.

[8] P. M. Wightman, M. A. Labrador, "Atarraya: A Simulation Tool to Teach and Research Topology Control Algorithms for Wireless Sensor Networks," in Proc. Simutools '09, 2009, Article No. 26.

[9] P. M. Wightman, M. A. Labrador, "A3: A Topology Construction Algorithm for Wireless Sensor Networks," in Proc. GLOBECOM, 2008, pp. 1-6.

[10] G. Pachnanda, K. Singh, L. Gangwar, "Comparative Analysis of A3, EECDS and KNEIGH Tree Protocols in Wireless Sensor Networks," International Journal of Electronics and Computer Science Engineering, vol. 2, no.3, pp. 987-991, 2013.

[11] H. Fornazier, A. Martin, S. Messner (2012) "Wireless Communication: Wi-Fi, Bluetooth, IEEE 802.15.4, DASH7," [Online]. Available: http://rose.eu.org/2012/wpcontent/uploads/2012/03/Wireless-communication.pdf

[12] R. C. Browning, E. A. Baker, J. A. Herron, R. Kram, "Effects of obesity and sex on the energetic cost and preferred speed of walking," Journal of Applied Physiology, vol. 100, no. 2, pp. 390-398, Feb. 2006. 